characteristics and results were transcribed and narratively synthesised into a pre-specified form. Each study was critically appraised by three researchers in accordance with internationally accepted criteria (STROBE, CONSORT, PRISMA).

Results Four relevant studies were identified and each assessed differing aspects of HPV vaccination and its association with sexual health. Vaccination was not a significant predictor of perceived vulnerability to cervical cancer $(p=0.601)$, intention to participate in HPV screening $(\mathrm{p}=0.521)$ or uptake of cervical screening $(\mathrm{p}=0.181)$. HPV vaccination was not a significant predictor of safer sexual behaviour $(\mathrm{p}=0.515)$ or consistent condom use $(\mathrm{p}=0.876)$.

Conclusion The results have proven inconclusive, as there is insufficient evidence to support or refute that HPV vaccination increases the risk of unsafe sexual behaviours. Notwithstanding, we observed a number of misconceptions regarding HPV, vaccination programs and cervical cancer screening. A positive HPV vaccination status contributed to a sense of complacency regarding the need for regular cervical cancer screening. Moreover, unvaccinated women were more likely to believe that HPV vaccine could be used as treatment for cervical cancer. As such these issues must be addressed in future research.

Disclosure of interest statement Nothing to declare

\section{P14.03 CHRONIC VULVAL PAIN/VULVODYNIA? PSYCHOSEXUAL ISSUES? TAKING A DIFFERENT APPROACH}

RA Hamilton*. University Hospital Barwon Health Geelong

\subsection{6/sextrans-2015-052270.515}

Vulvodynia was defined by the International Society for the Study of Vulvovaginal Disease (ISSVD) in 2003 as vulval discomfort, described as burning, stinging or irritation within the vulvovaginal region. This definition was to be in the absence of visible findings or identified clinical signs of neurological conditions. The condition can either be localised or general, with the discomfort experienced, being spontaneous or provoked by physical contact.

Other causative potential diagnosis include infections and dermatological issues. Importantly psychosexual concerns are to be ruled out as a causative nature. More often than not, the woman ends up on a long and arduous journey from General Practitioner, to Gynaecologist, onto a Physiotherapist and to a Psychologist. Too infrequently, she may be referred to a Sexologist.

Although a multidisciplinary approach is ideal to address the various often layered problems that gives rise to such a diagnosis of vulvodynia, from a retrospective clinical observation, it would be more beneficial to introduce the concept of a sexologist directly. Moreover, many women appear to be labelled with this diagnosis when in fact, it is often a complex psychosexual issue peppered with relationship difficulties.

The label of vulvodynia has the negative ability to cause increased emotional trauma, fear, anxiety and guilt. Subsequently, causing sexual problems rather than answers.

So who begins this discussion with the woman? Who opens the line of communication up to explore the layers? More importantly, why should we?

Disclosure of interest statement Nothing to Declare.

\section{P14.04 WHICH PSYCHOSOCIAL FACTORS ARE ASSOCIATED WITH POOR SEXUAL HEALTH OUTCOMES IN WOMEN OF REPRODUCTIVE AGE? A SYSTEMATIC REVIEW OF PROBABILITY SURVEYS}

${ }^{1,2} \mathrm{~N}$ Edelman, ${ }^{3} \mathrm{R}$ de Visser, ${ }^{4} \mathrm{C}$ Mercer, ${ }^{5} \mathrm{~L}$ McCabe, ${ }^{1,4} \mathrm{~J}$ Cassell ${ }^{*}$. ${ }^{1}$ Brighton \& Sussex Medical School; ${ }^{2}$ University of Brighton; ${ }^{3}$ University of Sussex; ${ }^{4}$ University College London; ${ }^{5}$ Leeds Teaching Hospital NHS Trust

\subsection{6/sextrans-2015-052270.516}

Introduction Interventions such as screening for sexually transmitted infections (STIs) and Contraceptive Advice and Supply (CAS) are increasingly provided in community settings, where populations are heterogeneous in risk. Identification of psychosocial determinants of poor sexual health may inform targeting strategies. We undertook a systematic review to identify psychosocial correlates of STI risk, risky sexual behaviours, unplanned pregnancy and abortion among women in the general population.

Methods We searched 7 databases (PsycInfo, Medline, ASSIA, Cochrane, CINAHL, Web of Science, Embase) to identify probability surveys and baseline longitudinal studies of women aged 16-44 reporting on associations between psychosocial factors and unplanned pregnancy, STI acquisition and sexual risk behaviours. We included studies from the European Union, USA, Canada, Australia, UK or New Zealand between 1/1994-1/2014.

Results Eleven papers were included. Unplanned pregnancy was associated with smoking, depression, relationship status and sexual debut $<16$ years. Abortion was associated with lack of parental closeness, leaving home at an early age, and abusive experiences. Non-use of contraception was associated with smoking, obesity, relationship status, sedentary lifestyles, and fatalistic attitudes to pregnancy. Condom non-use at first sex was associated with a partner $5+$ years older and with less stable partnerships. Multiple partnerships were associated with smoking, drug and alcohol use. STI diagnosis was associated with relationship break-up and young male partners.

Conclusion Relationship status and smoking were the factors most commonly reported to be associated with the adverse sexual health outcomes considered. Psychosocial variables may have utility in identifying women experiencing sexual risk behaviours in community settings, but STIs are too rare in the general population to be identified in this way. We plan to investigate the acceptability of psychosocial questions in targeting, and to explore whether unplanned pregnancy and STI acquisition are associated with different psychosocial factors.

Disclosure of interest statement This work is funded by England's National Institute for Health Research. No pharmaceutical grants or other funds were received in the development of this study.

\section{P14.05 SEXUAL CONTACT IS THE TRIGGER! WOMEN'S VIEWS AND EXPERIENCE OF THE CAUSES AND TRIGGERS OF BACTERIAL VAGINOSIS}

${ }^{1} \mathrm{~J} \mathrm{Bilardi}{ }^{*},{ }^{2} \mathrm{~S}$ Walker*${ }^{*}{ }^{2} \mathrm{M}$ Temple-Smith, ${ }^{2} \mathrm{R}$ McNair, ${ }^{3} \mathrm{~J}$ Mooney-Somers, ${ }^{1,4} \mathrm{C}$ Bellhouse, ${ }^{5} \mathrm{C}$ Fairley, ${ }^{5} \mathrm{M}$ Chen, ${ }^{5} \mathrm{C}$ Bradshaw. ${ }^{1}$ Monash University, Melbourne, Australia; ${ }^{2}$ The University of Melbourne, Melbourne, Australia; ${ }^{3}$ The University of Sydney, Sydney, Australia; ${ }^{4}$ Melbourne Sexual Health Centre, Melbourne, Australia; ${ }^{5}$ The University of Melbourne \& Melbourne Sexual Health Centre, Melbourne, Australia 